\title{
Activation of sphingosine-1-phosphate signalling as a potential underlying mechanism of the pleiotropic effects of statin therapy
}

\section{Abstract}

The mechanisms by which statins are beneficial are incompletely understood. While the lowering of low-density lipoprotein concentration is associated with regression of atherosclerosis, the observed benefit of statin therapy begins within months after its initiation, making regression an unlikely cause. Although LDL-C lowering is the main mechanism by which statin therapy reduces cardiovascular events, evidence suggests that at least some of the beneficial actions of statins may be mediated by their pleiotropic effects. Thus, statins may modulate the function of cardiovascular cells and key signalling proteins, including small Gproteins, to ultimately exert their pleiotropic effects. Sphingosine-1-phosphate (S1P) is a naturally occurring bioactive lysophospholipid that regulates diverse physiological functions in a variety of different organ systems. Within the cardiovascular system, S1P mediates cardioprotection following ischemia/reperfusion injury, anti-inflammatory response, improvement of endothelial function, increased mobilization and differentiation of endothelial progenitor cells, inhibition of oxidation, and anti-atherogenic and anti-thrombotic actions. Early evidence suggests that the pleiotropic effects of statins may be related to an increase in S1P signalling. This review focuses on S1P signalling as the potential mechanism underlying the pleiotropic effects of statins. An improved understanding of this mechanism may be vital for establishing the clinical relevance of statins and their importance in the treatment and prevention of coronary artery disease. Key points Several studies have demonstrated a benefit from lowering serum LDL-C with statins in patients with and without clinical evidence of CAD. These may be mediated by the pleiotropic effects of statins-the mechanisms of which are incompletely understood. Early evidence suggests that statins may increase S1P signalling pathways through upregulation of the expression of S1P receptors and an increase in plasma levels of S1P to ultimately exert their pleiotropic effects. Future clinical trials and basic science research aimed at the underlying mechanisms of the pleiotropic effects of statins should enlighten us to their relative clinical relevance and importance. 